\title{
PROPUESTA DE UNA ESTRUCTURA TRIDIMENSIONAL PARA EL DISEÑO DEL PRACTICUM DE PSICOPEDAGOGÍA
}

\author{
PROPOSAL OF A TRIDIMENSIONAL STRUCTURE TO DESIGN THE \\ PRACTICUM IN THE DEGREE OF PSYCHOPEDAGOGY
}

\author{
Sixto Cubo* y \\ Manuel Montanero**
}

\begin{abstract}
RESUMEN
El plan de estudios de la Titulación de Psicopedagogía contempla el Practicum como una materia de extraordinaria importancia. Tiene como objetivo fundamental desarrollar espacios empíricos en los que el alumnado pueda adquirir y desarrollar habilidades y competencias relacionadas con la intervención psicopedagógica. Presentamos en este artículo una propuesta de estructura tridimensional para el Practicum de Psicopedagogía basada en tres ejes vertebradores de la actividad del alumno: el contexto de desarrollo de la actividad práctica (educativo-institucional, sociocomunitario y organizacional), las áreas temáticas (procesos de enseñanza-aprendizaje, orientación y prevención y desarrollo) y las modalidades metodológicas para su diseño y desarrollo (valoración de programas, investigación-acción e investigación experimental, cuasiexperimental u observacional). Presentamos también, finalmente, algunas ideas relacionadas con el proceso de toma de decisiones del alumno en cuanto a cuál debe ser su Practicum, y un anexo que recoge algunas sugerencias relacionadas con los contenidos temáticos del Practicum de Psicopedagogía.
\end{abstract}

Palabras clave: practicum, psicopedagogía.

\section{ABSTRACT}

The syllabus of the degree of Psychopedagogy has the Practicum as a subject of great importance. It has as a main objective the development of empirical spaces where the students can acquire and develop skills and competences related to the psychopedagogical intervention. We present in this article a

* Doctor en Psicología por la Universidad Autónoma de Madrid. Profesor de la Universidad de Extremadura y Director de la División de Investigación y Prospección Educativa del I.C.E. de la Universidad de Extremadura. Líneas de investigación: métodos de investigación en Educación, análisis y tratamiento de datos, orientación profesional, sistemas expertos.

** Licenciado en Psicología y en Ciencias de la Educación. Doctor en Pedagogía. Profesor asociado de la Universidad de Extremadura. Líneas de investigación: modelos de orientación psicopedagógica y atención a la diversidad; didáctica de la ciencia y compresión de textos de ciencias sociales. 
proposal of a tridimensional structure for the Practicum of Psychopedagogy based on three vertebral ideas of the student's activity: the context of development of the practical activity (educational-institutional, sociocommunitary and organizational), the thematic areas (teaching-learning processes, guidance and prevention and development) and the methodological modalities for its design and development (programme evaluation, research-action and experimental research, quasiexperimental and observational). Finally,we also present some ideas related to the process of making decisions by the student regarding his/her Practicum, and an annex that gathers some suggestions related to the thematic contents of the Practicum of Psychopedagogy.

Key word: practicum, psychopedagogy.

\section{Contenidos temáticos del Practicum de Psicopedagogía}

La delimitación epistemológica y profesional de la Orientación Psicopedagógica es una de las cuestiones más problemáticas que afectan a la todavía incipiente titulación de Psicopedagogía. La orientación entendida como un «proceso de ayuda profesionalizada hacia la consecución de la promoción personal y la madurez social» (Pérez, 1987) supone la implicación de diferentes agentes educativos y sociales en diferentes aspectos del desarrollo del individuo. Se trata de un concepto multidimensional, cuya proximidad a otros como el de «asesoramiento», «formación»o al mismo concepto de «educación», hace que sea especialmente necesario especificar las finalidades, áreas y contextos profesionales que lo delimitan, así como los diferentes modelos desde los que se puede afrontar la intervención psicopedagógica. Desde luego, todo enmarcado en el objetivo general del Practicum de Psicopedagogía que es generar espacios empíricos de formación y de desarrollo profesional; espacios de confluencia de las estructuras teóricas que se han generado en las aulas con los conocimientos heurísticos que se producen como consecuencia del desarrollo de habilidades profesionales y/o del aprendizaje vicario producido en los diferentes contextos donde se desarrolla la actividad psicopedagógica.

El Plan de estudios y muy especialmente el Practicum de Psicopedagogía se encuentran en el núcleo de esta polémica. Los créditos de prácticas deberían ir dirigidos, en último término, a facilitar que el alumno conozca los objetivos, el plan de actuación, el contexto social y profesional de los centros, equipos, departamentos o servicios en los que se desarrolla la actividad psicopedagógica. Además, se pretende que los alumnos participen reflexivamente en dicha actividad a través de un proyecto de prácticas planificado y supervisado por los tutores del Practicum. No es de extrañar en consecuencia que no sólo los alumnos sino también los tutores nos veamos en ocasiones desbordados a la hora de plantear los posibles ámbitos y responsabilidades de actuación en dichos Proyectos. Para soslayar, en la medida de lo posible, este problema a la hora de limitar el contenido y estructura del proyecto de práctica debemos analizar al menos dos cuestiones previas: el contenido temático que se plantea en la intervención y la modalidad metodológica para el diseño, aplicación y evaluación de la misma.

Desde nuestro punto de vista, la elección del posible contenido temático requiere analizar en primer lugar las diferentes áreas sobre las que es posible planificar la intervención, dentro del contexto profesional en el que se encuadran aquellos centros, equipos, departamentos o servicios que acogen al alumno de prácticas.

Históricamente, la orientación psicopedagógica comenzó focalizándose sobre la orientación profesional, ampliada al ciclo vital y a su integración curricular desde el enfoque de 
la «educación para la carrera». Podríamos decir que la orientación profesional constituye el primer área de intervención psicopedagógica.

Posteriormente, la preocupación por mejorar la calidad del proceso de aprendizaje de los alumnos y combatir las dificultades recurrentes que se plantean en muchos de ellos amplió las demandas de la orientación en una doble vertiente: por un lado, hacia el asesoramiento de los docentes e instructores para mejorar los métodos y estrategias de enseñanza; por otro, hacia la intervención psicopedagógica para ayudar al propio alumno a desarrollar estrategias más efectivas. El resultado es un área suficientemente diferenciada que podemos denominar orientación sobre el proceso de enseñanza-aprendizaje.

Finalmente, a principios de los años setenta se desarrollan nuevos enfoques de la orientación como la denominada «educación para la salud» o la «educación psicológica», que incluyen nuevos ámbitos de intervención como las habilidades sociales, prevención del consumo de drogas, la educación en valores, el tiempo libre y otros ámbitos del desarrollo personal y social. Se trata pues de un área que, si bien presenta un claro «solapamiento» con objetivos y actuaciones anteriores, goza de entidad propia.

Por el contrario, a partir de la confluencia de la orientación en los procesos de enseñanza-aprendizaje con la Educación Especial, otros autores, como Álvarez y Bisquerra (1996), añaden como un cuarto ámbito «la atención a la diversidad». Desde nuestro punto de vista, es arriesgado tratar de delimitar aquí un conjunto de contenidos con una cierta independencia dentro del campo de la intervención psicopedagógica. La relevancia actual de la atención a las llamadas «necesidades educativas especiales» en la orientación no parece justificación suficiente para considerar la «atención a la diversidad» sino como un principio esencial de toda intervención en cualquiera de las áreas anteriores, una vez superadas las restricciones que históricamente han acompañado al modelo clínico. De ahí que hayamos decidido prescindir de su inclusión como un área más en la estructuración del Practicum.

En definitiva, hemos seleccionado tres áreas temáticas que agrupan, de forma no excluyente, los diferentes contenidos sobre los que puede versar el proyecto de Prácticas. Por otro lado, estamos plenamente de acuerdo con Bisquerra $(1996,1998)$ en la posibilidad de extrapolar tres grandes contextos en los que es posible localizar dichas áreas: el educativoinstitucional, el sociocomunitario y el organizacional.

La mayor parte de la profesionalización de la intervención psicopedagógica se concentra en instituciones directamente dirigidas a la educación formal. De hecho la mayoría de los alumnos de Psicopedagogía hacen sus Prácticas en Departamentos de Orientación de diversos centros (fundamentalmente de I.E.S.) y en los Equipos psicopedagógicos. La temática de estos proyectos de prácticas no puede equipararse sin embargo con la de otras practicas pedagógicas en el contexto educativo-institucional como las que realizan los alumnos de magisterio en Educación Infantil y Primaria o los alumnos del C.A.P. en Secundaria. Las prácticas de orientación, a diferencia de la estrictamente docente, suponen sobre todo una acción indirecta que repercute sobre la educación de los alumnos a través de la mejora y la innovación curricular, así como del asesoramiento de los profesores en su labor docente y tutorial. Ello supone, entre otras diferencias, que el contexto social de la actuación psicopedagógica venga definido por el trabajo colaborativo con otros profesionales de la educación, antes que por la actuación directa sobre los alumnos. En el cuadro 1, se esquematizan los principales objetivos y métodos de intervención psicopedagógica que podemos desarrollar en este contexto. 


\section{CUADRO 1: (Adaptado de Montanero 1997)}

\begin{tabular}{|c|c|c|c|c|}
\hline $\begin{array}{l}\text { Nivel de } \\
\text { acción }\end{array}$ & $\begin{array}{c}\text { ÁMBITO } \\
\text { (BOMEC, 13/5/96) }\end{array}$ & OBJETIVOS & METODOLOGÍA & RECURSOS \\
\hline \multirow[t]{3}{*}{$\begin{array}{l}\text { Personal } \\
\text { (Alumnos) }\end{array}$} & $\begin{array}{c}\text { Apoyo } \\
\text { psicopedagógico }\end{array}$ & $\begin{array}{l}\text { a) Evaluar y apoyar } \\
\text { psicopedagógicamente el } \\
\text { desarrollo personal y el proceso de } \\
E / A \text { del alumno, especialmente de } \\
\text { aquellos que presenten alguna DA. }\end{array}$ & $\begin{array}{c}\text { Evaluac.psicopedagógica } \\
\text { Prog. de desarrollo } \\
\text { socioafectivo* } \\
\text { Prog. de desarrollo } \\
\text { cognitivo* } \\
\text { Prog. de T.T.I. } \\
\text { Prog. de refuerzo } \\
\text { pedagógico* }\end{array}$ & \multirow[t]{3}{*}{$\begin{array}{c}\text { D. de } \\
\text { orientación } \\
\text { Aulas de apoyo } \\
\text { Equipos } \\
\text { generales }\end{array}$} \\
\hline & Apoyo al POAP & $\begin{array}{l}\text { b) Orientar el proceso de elección } \\
\text { vocacional de los alumnos }\end{array}$ & $\begin{array}{l}\text { Plan de Orientación } \\
\text { Académica y Profesional }\end{array}$ & \\
\hline & (Docente) & $\begin{array}{l}\text { (Optativas, PDC y programas } \\
\text { específicos de refuerzo pedagógico) }\end{array}$ & \multirow{2}{*}{$\begin{array}{l}\text { Elaboración de Proyectos } \\
\text { Pedagógicos } \\
\text { Proyectos de innovación }\end{array}$} & \\
\hline \multirow{3}{*}{$\begin{array}{l}\text { Institucional } \\
\text { (Profesores) }\end{array}$} & & $\begin{array}{l}\text { c) Asesorar el diseño y desarrollo } \\
\text { curricular, en la formación del } \\
\text { profesorado y en la investigación e } \\
\text { innovación didáctica }\end{array}$ & & \multirow{3}{*}{$\begin{array}{l}\text { D. didácticos } \\
\text { (CCP) } \\
\text { D. de } \\
\text { orientación } \\
\text { Equipos } \\
\text { específicos } \\
\text { Aulas de apoyo }\end{array}$} \\
\hline & $\begin{array}{l}\text { sesoramien } \\
\text { curricular }\end{array}$ & $\begin{array}{l}\text { d) Coordinar las acciones de atención } \\
\text { a la diversidad y la integración de } \\
\text { los alumnos con NEE }\end{array}$ & $\begin{array}{c}\text { Prog. de diversificación } \\
\text { curricular } \\
\text { Prog. de desarrollo } \\
\text { individual } \\
\text { Adaptaciones curriculares } \\
\text { Activ. de recuperación y } \\
\text { refuerzo }\end{array}$ & \\
\hline & Apoyo al PAT & $\begin{array}{l}\text { e) Coordinar la confección del PAT y } \\
\text { el desarrollo de las actividades de } \\
\text { tutoría y extraescolares }\end{array}$ & Plan de Acción Tutorial & \\
\hline \multirow{2}{*}{$\begin{array}{l}\text { Comunitario } \\
\text { (Comunidad } \\
\text { educativa) }\end{array}$} & \multirow[t]{2}{*}{ De cooperación } & $\begin{array}{l}\text { f) Informar y colaborar con las } \\
\text { familias y demás ámbitos } \\
\text { educativos, sociales y laborales }\end{array}$ & Escuela de Padres & \multirow{2}{*}{$\begin{array}{c}\text { D. de } \\
\text { orientación } \\
\text { Junta de } \\
\text { profesores } \\
\text { E. generales } \\
\text { APAs } \\
\text { Ayuntamiento }\end{array}$} \\
\hline & & $\begin{array}{l}\text { g) Conectar con los EOEP y demás } \\
\text { servicios de apoyo }\end{array}$ & $\begin{array}{l}\text { (Estrategias de } \\
\text { colaboración) }\end{array}$ & \\
\hline
\end{tabular}

La consideración de otros contextos al margen del estrictamente educativo es una cuestión polémica que ha planteado posiciones encontradas en diversos sectores. Esto explica la extendida descompensación entre el número de instituciones educativas que colaboran con el Practicum y aquellas otras enfocadas hacia la intervención comunitaria y organizacional.

El contexto sociocomunitario agrupa las actuaciones psicopedagógicas en medios comunitarios para ofertar servicios que faciliten la integración social del ciudadano y mejorar su calidad de vida. En este contexto el psicopedagogo tiene unas áreas de intervención realmente amplias, desde luego no relacionadas con los modelos tradicionales que asocian la actividad psicopedagógica únicamente con las actividades educativas formales. En este sentido, y trabajando para la comunidad, y con los recursos que la comunidad pone a disposición de numero- 
sos profesionales para desarrollar actividades diversas, el psicopedagogo diseña, aplica y evalúa planes de formación relacionados con, por ejemplo, las Universidades Populares; realiza evaluación de programas para instituciones de carácter público o privado; diseña programas de orientación, junto con los interlocutores sociales, para establecer itinerarios de encuentro con el empleo; colabora con otros profesionales para la eliminación de las barreras psicológicas que impiden el acceso al mercado laboral; desarrolla programas para la eliminación de las barreras, no sólo arquitectónicas, sino también actitudinales y comportamentales que limitan las posibilidades de integración social y laboral de las personas discapacitadas, etc.

Todo este conjunto de actividades, que como ejemplos hemos mencionado, junto con unos procedimientos metodológicos depurados que más adelante comentaremos, y que garantizan científicamente tanto los procesos como los resultados, nos permite ofrecer a nuestros alumnos una perspectiva más enriquecedora, más global, más real en una palabra, de qué es un psicopedagogo, qué es lo que la sociedad demanda del psicopedagogo y qué es lo que la Psicopedagogía puede ofrecer a la sociedad.

Por último, en el contexto organizacional, el psicopedagogo aporta recursos para mejorar la actividad laboral y potenciar la satisfacción laboral y el desarrollo personal. Al igual que en el contexto sociocomunitario, las actuaciones psicopedagógicas deben ser percibidas desde un marco de actuación interdisciplinar. Esto es así porque en estos contextos el psicopedagogo colabora con otros profesionales para el logro de objetivos comunes expresados a nivel de unidad, de departamento, de sección, de servicio, o de la empresa analizada globalmente. En estos contextos el psicopedagogo trabaja no sólo para el desarrollo de habilidades, de conocimientos o de competencias expresados estos objetivos a través de programas de formación, sino también en el diseño y aplicación de programas dirigidos al desarrollo de planes de promoción interna, y de desarrollo personal y profesional dentro de la empresa. De esta manera, y con el desarrollo de estas acciones que como ejemplo hemos mencionado, el psicopedagogo interviene también, colaborando con otros profesionales, en la prevención de la aparición de algunos síndromes psicopatológicos de especial importancia en el mundo de las organizaciones, tales como el síndrome de Burnout, o el efecto «mobbing ${ }^{1} »$.

De una manera especial hemos de nombrar, por la también especial relevancia que tiene en el mundo de la empresa, y por la importancia de estas actividades en las expectativas que podemos elaborar en relación con el desarrollo de la profesión de psicopedagogo, a las actividades relacionadas con la Formación de Formadores. En este sentido creemos que el psicopedagogo es un profesional especialmente cualificado para diseñar y aplicar programas dirigidos al desarrollo de las habilidades de los docentes que participan en la formación continua, así como para el diseño de las acciones de evaluación de los planes de formación dentro de las empresas.

Uno de los problemas aún no resueltos, principalmente debido a la tradición pedagógica de las instituciones donde se imparten los planes de estudios de Psicopedagogía, es la no existencia de canales de comunicación adecuados con el mundo de la empresa. A pesar de que por ejemplo en algunas instituciones públicas de carácter estatal, el psicopedagogo está contemplado en los perfiles profesionales relacionados con el desarrollo de actividades dirigidas al aumento de la ocupabilidad de las personas desempleadas, y de esta manera participan activamente desde hace algún tiempo en el desarrollo de actuaciones dirigidas a favore-

\footnotetext{
1 Nos referimos al terror psicológico en el puesto de trabajo como consecuencia del desamparo generado por el aislamiento autoimpuesto, el producido por los compañeros, o el impuesto por los superiores.
} 
cer el encuadramiento adecuado de los demandantes de empleo, o a potenciar las habilidades relacionadas con la búsqueda activa de empleo, todavía tenemos problemas para ubicar correctamente a los alumnos que desean desarrollarse profesionalmente en el mundo de las organizaciones. Creemos, en este sentido, que es importante establecer canales oficiales de comunicación que institucionalicen estas actividades psicopedagógicas.

\section{Modalidades del proyecto de prácticas}

Estos presupuestos nos permiten clasificar los contenidos temáticos del Practicum cruzando los anteriores criterios (como hemos realizado, de forma exhaustiva, en el Anexo de esta comunicación). De todas formas, creemos necesario añadir a toda esta reflexión una tercera dimensión inherente a cualquier actuación psicopedagógica. Una vez delimitado el contenido de las Prácticas, el alumno demanda sobre todo una ayuda sistemática que le ayude a diseñar e implementar el Proyecto de Prácticas. En este sentido, podemos organizar el Proyecto en torno a dos grandes modalidades metodológicas, con características claramente diferentes tanto en su planificación y desarrollo como en la presentación escrita como parte de la Memoria final que debe elaborar el alumno: los proyectos de evaluación e intervención y los proyectos de investigación psicopedagógica.

Dentro de la modalidad de evaluación e intervención psicopedagógica, podemos distinguir tres tipos de proyectos. Los que se basan en el diseño y/o desarrollo de programas de evaluación y desarrollo personal y madurativo, agrupan diversos proyectos centrados en el estudio de casos, la evaluación psicológica de problemas específicos y la elaboración de Programas de Desarrollo Individual. El carácter generalmente asistencial y reactivo de los mismos (desarrollados generalmente de forma paralela al currículo) los convierte en un tipo de intervención típica del modelo clínico, menos extendida en el contexto educativo a partir del modelo psicopedagógico o «educativo-constructivo» (Solé, 1997) apoyado por la Reforma. En el cuadro 2 hemos sintetizado los principales apartados que debería recoger un Proyecto de esta modalidad.

\section{CUADRO 2: Programas de evaluación y desarrollo personal y madurativo}

- Recogida de información previa.
- Formulación de hipótesis y deducción de enunciados verificables.
- Resultastación.
- Formulación de hipótesis funcionales.
- Diseño y aplicación de la intervención (P.D.I.).
- Contrastación de hipótesis y valoración del tratamiento.
- Resultados y seguimiento.

Por otro lado, el M.E.C. sintetiza en el Documento Individual de Adaptaciones Curriculares (D.I.A.C.) un conjunto de orientaciones (véase nuestra propuesta en el cuadro 3) para diseñar la evaluación psicopedagógica y la intervención en contextos curriculares, para aquellos alumnos que presentan necesidades educativas especiales (Orden de 14 de febrero de 1996, Art. $4^{\circ}$, sobre la evaluación de los alumnos con necesidades educativas especiales que 


\section{CUADRO 3: Adaptaciones curriculares (D.I.A.C.)}

- Recogida de información (síntesis de la evaluación psicopedagógica): historia escolar y de aprendizaje; estilo de aprendizaje; intereses y motivaciones; contexto escolar y sociofamiliar; nivel de competencia curricular.

- Necesidades Educativas Especiales .

- Propuesta de adaptación curricular (Ciclo/U.D.): de acceso; objetivos y criterios de evaluación; contenidos; actividades y metodología.

- Estrategias de colaboración con las familias.

- Valoración y seguimiento.

cursan las enseñanzas de régimen general). La relevancia de este tipo de demandas para el orientador (y para el alumno de prácticas) justifica, a nuestro parecer, su consideración como una modalidad independiente, a pesar de ser exclusiva del contexto educativo-institucional.

Por último, el diseño y desarrollo de programa de orientación en cualquiera de los contextos debería recoger los apartados que aparecen en el cuadro 4. En algunos casos, la dificultad mayor reside en la coordinación de muy diversos programas en «macroprogramas» integrados (que hemos denominado «planes») a lo largo de períodos extensos de tiempo, Sería por ejemplo el caso de una prácticas dirigidas a la elaboración o revisión de un Plan de Acción Tutorial.

\section{CUADRO 4: Programas y planes de orientación}

- Planificación previa de necesidades y recursos (recogida de información, justificación...).

- Objetivos.

- Componentes/contenidos, estrategias/actividades y recursos/materiales

- Fases, temporización y metodología de implementación: movilización, entrada a la comunidad, implantación, mantenimiento del programa, institucionalización, diseminación...

- Evaluación.

Por su parte, la investigación psicopedagógica puede plantearse desde al menos tres tipos de proyectos. La valoración de programas (Fernández Ballesteros, 1990) se basa en la concreción de indicadores e instrumentos que permitan evaluar la eficacia de un "paquete» de intervención psicopedagógica ya diseñado, o de un proyecto curricular en función de una serie de objetivos específicos (ver cuadro 5).

\section{CUADRO 5: Valoración de programas de intervención psicopedagógica y proyectos curriculares}

- Descripción del programa/proyecto.

- Objetivos y dimensiones de la valoración.

- Selección de unidades, aspectos o tratamientos del programa a valorar.

- Selección de indicadores y operaciones.

- Fases y procedimiento de evaluación.

- Análisis de datos.

- Discusión y generalización de resultados. 
Por su parte, la investigación sobre determinados fenómenos psicopedagógicos en cualquiera de los contextos profesionales de la orientación puede plantearse desde dos tipos de enfoques según se asuma un enfoque metodológico más próximo a la perspectiva cuantitativa o a la cualitativa. Desde el paradigma de la investigación-acción (véase por ejemplo, Blázquez, 1991) los apartados del proyecto (ver cuadro 6) deberían reflejar cómo se contempla la participación investigadora de los diferentes agentes que participan en la propia intervención psicopedagógica, así como las oportunas triangulaciones que ofrezcan un mínimo de objetividad, especialmente cuando estas evaluaciones tienen un marcado carácter cualitativo.

\section{CUADRO 6: Investigación-acción}

- Planteamiento inicial, participativo o colaborativo, del proyecto de innovación.

- Fases y procedimiento de evaluación (de la movilización, implantación e institucionalización del proyecto...).

- Elección de sujetos y técnicas de recogida de datos (previsión de triangulaciones).

- Reflexión crítica (colaborativa): interpretación de resultados y replanteamiento de estrategias.

Por el contrario, un proyecto de investigación vinculado a la metodología cuantitativa se basaría, en el caso de investigaciones experimentales o cuasiexperimentales, en la formulación de una hipótesis de trabajo en la que queden definidas las variables independientes y las dependientes, así como una dirección en cuanto a los resultados que esperamos encontrar. Haríamos hincapié también en la operativización de esas variables, en las técnicas de control para garantizar tanto la validez interna como la externa y en los procedimientos de análisis de datos a desarrollar, tanto en su apartado descriptivo como inferencial (ver cuadro 7).

\section{CUADRO 7: Investigación observacional o experimental}

- Planteamiento del problema.
- Formulación de hipótesis.
- Definición operativa de variables.
- Control de variables extrañas.
- Muestreo.
- Diseño y procedimiento.
Análisis de datos.

\section{Conclusiones}

Nuestra propuesta de organización del Practicum de Psicopedagogía se asienta por tanto en una estructura tridimensional en la que podemos distinguir tres vectores: las áreas y contextos profesionales (que permiten acotar los diversos contenidos temáticos) y las modalidades metodológicas para su diseño y desarrollo (ver gráfico 1). 


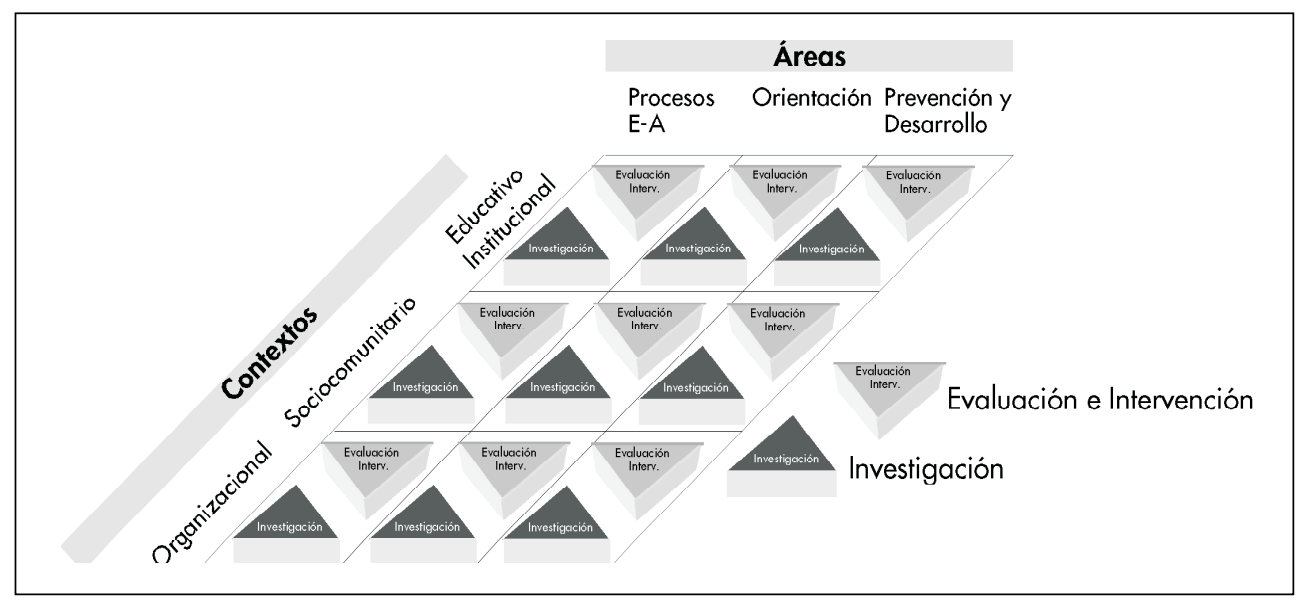

\section{GRÁFICO 1}

Sobre este modelo obtenemos 18 tipos de proyectos de prácticas (que se convierten en 54, si consideramos cada modalidad específica de proyecto), con una suficiente consistencia para abordar una reflexión científica sobre la experiencia obtenida. Si tuviéramos en cuenta la clasificación de contenidos temáticos por contexto y área (ver anexo), obtendríamos en torno a 400 diferentes posibilidades que prácticamente agotan toda la casuística de proyectos del Practicum de Psicopedagogía. Desde luego creemos, como ya hemos comentado anteriormente, que no todas estas actividades sugeridas forman parte de un perfil exclusivamente asignado al profesional de la Psicopedagogía, más bien creemos que el trabajo en muchas de estas actividades, de estas Áreas de desarrollo profesional, es un trabajo colaborativo, es una propuesta de actuación basada en modelos de afrontamiento interdisciplinar de la realidad.

Un tema que nos preocupa bastante dada la complejidad de la estructura generada es el proceso de toma de decisiones por parte del alumno. Creemos que es necesario provocar reflexión acerca de las implicaciones de ese proceso de toma de decisiones, es decir, el alumno debe posicionarse en relación a los siguientes elementos que configurarán su elección:

1. Los imperativos de la institución y la realidad social y profesional que acoge al alumno en prácticas (sentimos que este sea el primer elemento propuesto, pero los principios de realidad y viabilidad se imponen).

2. La naturaleza del trabajo a desarrollar: vinculado a Psicopedagogía Básica o a Psicopedagogía Aplicada. En el primer caso optaremos por un trabajo relacionado con la investigación psicopedagógica, en el segundo por uno de evaluación-intervención. Supongamos que el alumno decide trabajar en Psicopedagogía Aplicada, la estructura queda de la manera representada en el gráfico 2 .

3. Los contextos de desarrollo profesional: las motivaciones, expectativas y experiencias previas deberían inclinar al alumno a optar por un contexto Educativo-Institucional, Sociocomunitario u Organizacional. Supongamos, por ejemplo, que el alumno opta por trabajar en un contexto Educativo-Institucional, la estructura progresa como muestra el gráfico 3. 


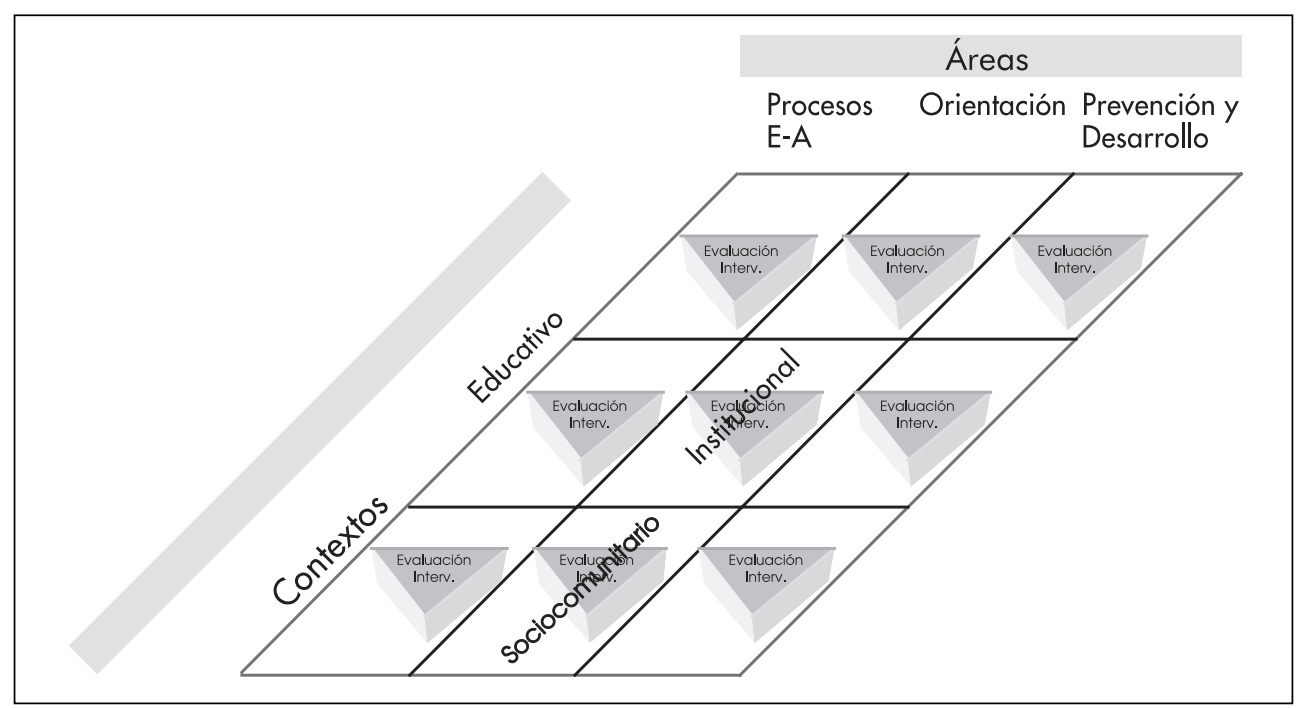

GRÁFICO 2

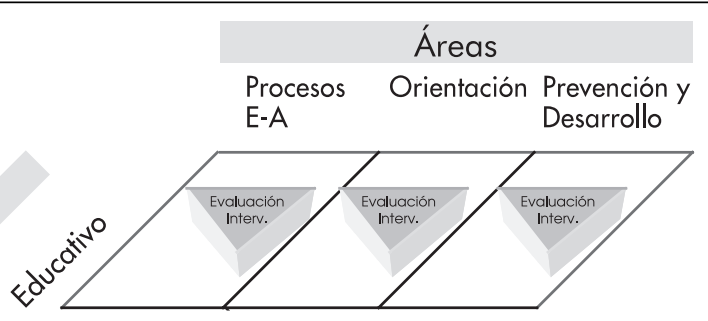

GRÁFICO 3

4. Áreas temáticas: por último la estructura está bastante más despejada, el alumno sólo debe decidir si desea trabajar en Procesos de Enseñanza-Aprendizaje, en Orientación o en Prevención y Desarrollo. Supongamos que su orientación se dirige hacia la Prevención y el Desarrollo, la elección está hecha (ver figura 4).

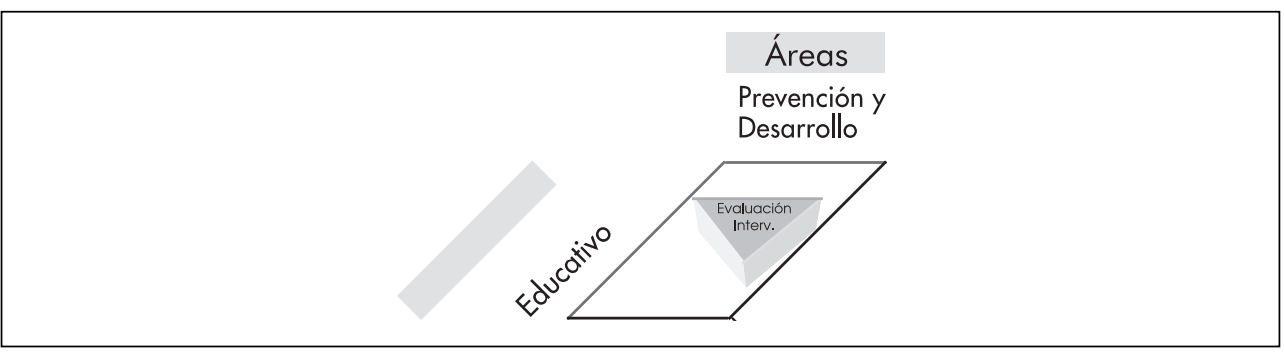

GRÁFICO 4 
No creemos que todo este análisis sea en absoluto gratuito. Por bien que se plantee el proyecto de prácticas, éste no dejará de ser una experiencia relativamente puntual en el vasto campo profesional que hemos tratado de delimitar, difícilmente abarcable para los 9 créditos del Practicum. Es necesario que el trabajo del alumno este presidido por una reflexión inicial que le ayude a localizar y comparar la institución y la intervención elegida en relación a otros tipos de contenidos y métodos posibles.

Por otro lado, el análisis de los componentes de cada tipo de proyecto puede constituir un apoyo eficaz para que, con ayuda de los tutores, el alumno aplique con mayor rigor científico los procedimientos metodológicos que intervienen en el diseño, evaluación e investigación psicopedagógica, así como en el informe escrito de los resultados obtenidos. Ello debe traducirse en una evaluación, compartida por los dos tutores, que se fundamente además de en la asistencia y el nivel de colaboración en las diferentes actividades desarrolladas en otros criterios relativos a la fundamentación teórica y contextual de la intervención elegida y el formato y rigor metodológico con que se ha diseñado tanto el Proyecto como la Memoria final.

\section{Bibliografía seleccionada para el Practicum}

Álvarez, M. y Bisquerra, R. (coord.) (1996): Manual de Orientación y Tutoría. Barcelona: Praxis. Anguera, M. T. (1983): Manual de prácticas de observación. México: Trillas.

ANGUeRA, M. T. (1991): Metodología observacional en la investigación psicológica. Barcelona: P.P.U.

Anguera, M. T. et al. (1995): Métodos de investigación en Psicología. Madrid: Síntesis.

Anton, K. (1990): Práctica de la dinámica de grupos. Barcelona: Herder.

ARCE, C. (1994): Técnicas de construcción de escalas psicológicas. Madrid: Síntesis Psicología.

BISQUERRA, R. (1996): Orígenes y desarrollo de la orientación psicopedagógica. Madrid: Narcea

BISQUERRA, R. (coord.) (1998): Modelos de orientación e intervención psicopedagógica. Barcelona: Praxis.

BLÁZquez, F. (1991): La investigación-acción. Métodos y técnicas de investigación cualitativa, en Sáenz, O. (coord.) Prácticas de enseñanza. Alcoy: Marfil.

Brueckner, L. J. y Bond, G. L. (1992): Diagnóstico y tratamiento de las dificultades en el aprendizaje. Madrid: Rialp.

Buendía Eisman, L. (1997): Métodos de investigación en psicopedagogía. Madrid: McGraw Hill.

CANDEL, I. (dir.) (1993): Programas de atención temprana. Madrid: CEPE.

Comeche Moreno, M. I., Díaz Garcoa, M. I. et al. (1995): Cuestionarios, inventarios y escalas. Ansiedad, depresión y habilidades sociales. Madrid: UNED-Fundación Universidad-Empresa.

Costa, M. y LóPEz, E. (1980): Salud Comunitaria. Barcelona: Martínez Roca.

Costa, M. y LóPez, E. (1991): Manual para el educador social. Vol. 1: Habilidades de comunicación en la relación de ayuda. Vol. 2: Afrontando situaciones. Madrid: M. Asuntos Sociales.

Del Pozo, P. (1993): Formación de Formadores. Madrid: Eudema.

Fermoso, P. (1994): Pedagogía social. Barcelona: Herder.

Fernández Ballesteros, R. (1990): Introducción a la evaluación Psicológica. Madrid: Pirámide 
García Vidal, J. (1990): Manual para la confección de programas de desarrollo individual. Madrid: EOS.

GARCía VIDAL, J. (1993): Guía para realizar adaptaciones curriculares. Madrid: EOS.

Gil Llario, M. D., M. D. LACER et al. (1992): Comparación de dos programas para el entrenamiento de las habilidades sociales de los escolares rechazados y olvidados. Valencia: Promolibro-Cinteco.

LeÓN, O. G. y Montero, I. (1993): Diseño de investigaciones: Introducción a la lógica de la investigación en Psicología y Educación. Madrid: McGraw-Hill.

Méndez Alcolea, A. y CAlvo, A. (1996): Técnicas para evaluar la competencia curricular. Madrid: Escuela Española.

Monereo, C. y Solé, I. (coord.) (1997): El asesoramiento psicopedagógico: una perspectiva profesional y constructivista. Madrid: Alianza.

Peiró, J. M. y Ramos, J. (1994): Intervención psicosocial en las organizaciones. Barcelona: PPU.

Peiró, J.M. y Prieto, F. (1996): Tratado de Psicología del Trabajo. Madrid: Síntesis.

PÉREZ, A. (1987): Tutoría y orientación. Madrid: ICE de la Universidad Complutense.

SCHEIN. E. H. (1990): Consultoría de procesos. Su papel en el desarrollo organizacional. Vol. 1 y 2. México: Addison-Wesley Iberoamericana. 


\section{ANEXO \\ Contenidos temáticos del Practicum}

\section{Contexto educativo-institucional}

\subsection{Orientación de los procesos de E/A}

- Asesoramiento o revisión del Proyecto Educativo de Centro, en función de las necesidades detectadas y de los principios psicopedagógicos y organizativos decididos por la comunidad educativa.

- Asesoramiento y colaboración con la Comisión de Coordinación Pedagógica y con el profesorado para el diseño, desarrollo o revisión del Proyecto Curricular de centro (directrices generales, diseños curriculares de área, programa base de diversificación curricular, plan de acción tutorial...).

- Diseño y desarrollo de Proyectos de Innovación didáctica con el fin de contribuir a la optimización de los procesos de enseñanza y aprendizaje, especialmente en el marco de las programaciones de aula.

- Evaluación psicopedagógica de alumnos con NEE y asesoramiento y colaboración con el profesorado en la planificación, desarrollo y evaluación de adaptaciones curriculares individuales de ciclo y de unidad didáctica.

- Apoyo psicopedagógico a alumnado que presente problemas específicos de aprendizaje, mediante el diseño, desarrollo y evaluación de actividades de T.T.I., programas de desarrollo cognitivo, programas de refuerzo pedagógico...

- Intervención psicopedagógica para la optimización del aprendizaje y el desarrollo en grupos de alumnos procedentes de sectores social y culturalmente desfavorecidos: evaluación de las necesidades educativas especiales, asesoramiento y colaboración con el profesorado en el diseño, desarrollo y evaluación de programas de intervención en el aula.

- Valoración del Plan de Formación, y coordinación de necesidades de los profesores con propuestas de la Administración, nivel de satisfacción del profesorado, etc.

\subsection{Orientación profesional}

- Diseño y valoración de un Programa de Orientación Académica y Profesional (P.O.A.P.).

- Diseño y valoración de actividades de toma de decisiones y orientación vocacional.

- Asesoramiento y colaboración con el profesorado en la integración curricular de contenidos relacionados con el mundo profesional.

- Diseño y valoración de actividades de orientación para la transición a la vida activa.

- Diseño y valoración de actividades de orientación para la igualdad entre los sexos. 


\subsection{Orientación para la prevención y el desarrollo}

- Diseño, desarrollo y valoración de programas de atención temprana.

- Evaluación e intervención de problemas específicos que afectan al desarrollo personal y madurativo de un alumno.

- Desarrollo de Proyectos de innovación educativa con el fin de desarrollar habilidades de prevención y desarrollo personal.

- Diseño de actividades de tutoría y asesoramiento de las Escuelas de Padres.

- Intervención psicopedagógica para ayudar al alumnado que presente problemas de desarrollo socioafectivo: integración social, autoestima, habilidades sociales...

- Intervención psicopedagógica para prevenir problemas propios del contexto social y profesional del docente, mediante habilidades de comunicación, trabajo en equipo, afrontamiento de situaciones de estrés...

- Diseño, desarrollo y valoración de programas de educación en valores.

- Intervención psicopedagógica para favorecer la educación intercultural y la integración social de todos los alumnos en aulas donde convivan personas o grupos procedentes de etnias o culturas diferentes.

- Intervención psicopedagógica para favorecer una educación no sexista.

- Asesoramiento sobre el tratamiento curricular de los temas transversales (educación para la paz, educación para la salud, educación para el consumo, educación ambiental).

- Asesoramiento y colaboración en actividades «extraescolares» dentro del centro educativo.

Posibles instituciones colaboradoras

- Departamentos de Orientación (Centros de Primaria e I.E.S.).

- E.O.E.P. (Generales, específicos y de atención temprana).

- Centros específicos de Educación Especial (APNABA, ASPACEBA, APROSUBA, APMEX, ADABA, Colegio de la Luz; Asociación Síndrome Down...).

- C.P.R.

- Centros de Menores.

\section{Contexto sociocomunitario}

\section{1. Área de orientación de los procesos de E/A}

- Intervención psicopedagógica en actividades destinadas a los niños todavía no escolarizados o que necesitan ser atendidos en el horario extraescolar: grupos de juego, ludotecas, hogares infantiles...

- Apoyo psicopedagógico en actividades educativas «no formales» desarrolladas en centros culturales, asociaciones, O.N.G.s, hospitales, prisiones, etc. con diferentes objetivos: alfabetización, educación de adultos, acceso a la Universidad... 
- Apoyo psicopedagógico de alumnos con deprivación sociocultural: evaluación, asesoramiento y colaboración con el profesorado en el diseño, desarrollo y evaluación de programas de intervención en contextos sociocomunitarios.

- Formación de formadores.

- Desarrollo de programas de evaluación de programas.

\subsection{Orientación profesional}

- Diseño, aplicación y valoración de programas de formación y orientación laboral (F.O.L.) dirigidos a individuos que han abandonado ya el sistema educativo y aún no se han integrado en el mercado de trabajo.

- Desarrollo de programas para la elaboración de itinerarios de encuentros con el empleo.

- Desarrollo de programas de información profesional.

- Desarrollo de programas para mejorar las habilidades de búsqueda de empleo.

- Asesoramiento, diseño y valoración de programas para la integración laboral de discapacitados.

\subsection{Orientación para la prevención y el desarrollo}

- Diseño, aplicación y valoración de programas relacionados con las actividades de tiempo libre.

- Diseño de actividades y asesoramiento psicopedagógico de las Escuelas de Padres.

- Intervención psicopedagógica en «educación permanente», medioambiental, de educación para la salud, etc., en contextos «no formales», como centros culturales, asociaciones, O.N.G.s, hospitales, prisiones... Asesoramiento y colaboración con el profesorado en el diseño, desarrollo y evaluación de los programas.

- Intervención psicopedagógica en problemas de drogodependencia.

- Diseño y desarrollo de programas de reeducación con familias desestructuradas.

- Asesoramiento en programas educativos en diversos medios de comunicación.

- Diseño y desarrollo de programas de animación sociocultural y de «educación de calle».

- Diseños, aplicación y evaluación de programas para la eliminación de las barreras psicológicas relacionadas con la inserción laboral.

Posibles instituciones colaboradoras

- CÁRITAS.

- ONCE.

- INSERSO.

- Hogar infantil de la Diputación Provincial.

- Centros de Menores. 
- Hospitales.

- Garantía Social.

- Escuelas Taller y Casa de Oficio.

- Hogares de ancianos.

- Centros penitenciarios.

- Asociaciones de vecinos.

- Casas de mujeres.

- Teléfono de la esperanza.

- Agentes Sociales.

- Asociaciones de ayuda al discapacitado: APROSUBA, FASEX, etc.

- A.P.A.s.

\section{Contexto organizacional}

\section{1. Área de orientación de los procesos de E/A}

- Asesoramiento, diseño y valoración de programas para la formación permanente en la empresa.

- Asesoramiento, diseño y desarrollo de productos para el mercado educativo.

- Formación de formadores.

- Desarrollo de programas de evaluación de programas.

\subsection{Orientación profesional}

- Asesoramiento, diseño y valoración de programas para la eliminación de las barreras que impiden la integración laboral de discapacitados en la empresa.

- Asesoramiento para la reorientación de la carrera de trabajadores en transición de un puesto de trabajo a otro («outplacement»).

- Selección, planificación y desarrollo de recursos humanos.

\subsection{Orientación para la prevención y el desarrollo}

- Asesoramiento, diseño y valoración de programas de salud laboral.

- Programas de prevención y afrontamiento del estrés.

- Programas para la prevención de conflictos interpersonales en las relaciones laborales, para el desarrollo de habilidades sociales, trabajo en equipo y liderazgo.

Posibles instituciones colaboradoras

- Fundación Laboral de la Construcción.

- O.N.C.E. 
- Servicio de Psicología del Ejército.

- Dirección General de Empleo de la Junta de Extremadura.

- Dirección General de Trabajo de la Junta de Extremadura.

- Agentes Sociales.

- INEM.

- Escuelas Taller y Casas de Oficios.

- Equipos de Promoción Laboral de la Diputación de Badajoz.

Fecha de recepción: 17-2-00

Fecha de revisión: 27-3-00

Fecha de aceptación: 11-4-00 